\title{
Emergência de flebotomíneos (Diptera: Psychodidae) em chão de floresta de terra firme na Âmazônia Central do Brasil: Uso de um modelo modificado de armadilha de emergência
}

\author{
Ronildo Baiatone ALENCAR ${ }^{1}$
}

\section{RESUMO}

Informaçōes acerca de potenciais criadouros naturais de flebotomíneos sempre foram de fundamental interesse epidemiológico. Contudo, são poucas as informaçōes advindas dos diversos estudos realizados até o momento. Isto se deve principalmente às dificuldades de localização e extração dos imaturos que se desenvolvem no solo e matéria orgânica do chão de florestas. No presente estudo o modelo modificado de armadilha de emergência foi testado na Vila do Pitinga, município de Presidente Figueiredo, Estado do Amazonas, a fim de localizar potenciais criadouros naturais. Vinte e sete indivíduos de nove espécies (Lutzomyia umbratilis, $L$. monstruosa, L. ayrozai, L. anduzei, L. trichopyga, L. davisi, L. geniculata, L. georgii e L. saulensis) foram coletados. Lutzomyia umbratilis foi a espécie com maior número de indivíduos, 10 , representando $37,1 \%$ do total. A produção de flebotomíneos foi estimada em 2,2 flebotomíneos por $100 \mathrm{~m}^{2}$ por dia. Em setembro, mês com maior número de indivíduos, esta produção foi de 5,8.

\section{PALAVRAS-CHAVE}

Flebotomíneo, Lutzomyia, armadilha de emergência, criadouros naturais, Amazônia Central.

\section{Emergence of phlebotomine sandflies (Diptera: Psychodidade) in non- flooded forest floor in Central Amazon, Brazil: A modified emergence trap model}

\section{ABSTRACT}

Information concerning the potential natural breeding sites of phlebotomine sandflies are of high epidemiological importance. However, few studies have been conducted on the subject. This is due especially to the difficulties in finding as well as extracting immature sandflies that develop in the soil and organic matter of the forest floor. In the present study, a modified emergence trap model was tested in order to find potential breeding sites. This model was tested in the Pitinga Village, situated in the Presidente Figueredo municipality, in the State of Amazonas. Twenty-seven individuals belonging to nine species (Lutzomyia umbratilis, L. monstruosa, L. ayrozai, L. anduzei, L. trichopyga, L. davisi, L. geniculata, L. georgii $e$ L. saulensis.) were collected. Lutzomyia umbratilis showed the highest number of individuals (37.1\%) of all species captured in the area. The phlebotomine productivity was estimated as 2.2 sandflies per $100 \mathrm{~m}^{2} /$ day. September showed the highest density of individuals, with a productivity of 5.8 .

\section{KEY WORDS}

Phlebotomine sandflies, Lutzomyia, emergence trap, natural breeding sites, Central Amazon.

\footnotetext{
1 Instituto Nacional de Pesquisas da Amazônia, Coordenação de Pesquisas em Ciências da Saúde - CPCS. e-mail: ronildo@inpa.gov.br
} 


\section{INTRODUÇÃO}

A busca por criadouros naturais de flebotomíneos sempre foi de fundamental interesse epidemiológico. Entretanto, até o presente momento, a grande maioria dos trabalhos com criadouros naturais demonstra escassos resultados quanto ao número de imaturos encontrados. Este baixo rendimento, muitas vezes, está diretamente relacionado às dificuldades de extração destes imaturos das amostras de solo e matéria orgânica onde normalmente são encontrados. Feliciangeli (2004), em uma recente revisão sobre criadouros naturais de flebotomíneos, cita as várias técnicas utilizadas em diversos estudos para a busca direta (busca por imaturos) e indireta (busca por adultos recém emergidos) de potenciais criadouros destes insetos. Entre as diversas técnicas citadas por esta autora, a armadilha de emergência é a técnica mais utilizada para busca indireta. Rutledge \& Ellenwood (1975a), usando esta técnica no Panamá, apontaram as camadas superficiais do chão da floresta como sendo os criadouros preferenciais de algumas espécies. Aplicando essa mesma técnica, Arias \& Freitas (1982) registraram a ocorrência de 16 espécies de flebotomíneos, todas do gênero Lutzomyia em solo de floresta na Amazônia Central. Utilizando um modelo de armadilha de emergência de plástico, modificado a partir de Bettini et al. (1986), em área endêmica de leishmaniose no Estado de São Paulo, Casanova (2001) registrou o encontro de 73 espécimes de flebotomíneos distribuídos entre três espécies do gênero Lutzomyia.

O presente estudo teve como principal objetivo testar um modelo modificado de armadilha de emergência para captura de adultos de flebotomíneos, cujos imaturos se desenvolvem no chão da floresta.

\section{MATERIAL E MÉTODOS}

\section{DESCRIÇÃO DA ARMADILHA}

A armadilha de emergência utilizada neste trabalho foi criada a partir do modelo de foto-ecletor utilizado por Penny \& Arias (1982). É uma armadilha leve e desmontável composta de duas partes principais: uma inferior feita de armação metálica e rede de tecido semitransparente de náilon, de estrutura piramidal com base de 50 por $50 \mathrm{~cm}$ e ápice truncado $(10 \times 10 \mathrm{~cm})$, e altura de $45 \mathrm{~cm}$, e uma superior, formada por um aparato de 25 $\mathrm{cm}$ de altura composto por dois potes plásticos de Nalgene ${ }^{\circledR} \mathrm{e}$ um funil (Figura 1). A armação metálica é formada por eixos de ferro galvanizado com $2 \mathrm{~mm}$ de espessura, que são encaixados em cantoneiras de cobre trifurcadas (Figura 2). A rede, fixada a esta armação metálica por meio de barbantes, possui na parte superior uma manga de $15 \mathrm{~cm}$ de comprimento e na inferior abas de $20 \mathrm{~cm}$ feitas com tecido de "napa". Estas abas, dobradas para o lado externo da base da armadilha, além de evitar o contato direto do tecido da rede e dos eixos de ferro com o chão da floresta, auxiliam também na fixação da armadilha no substrato, já que sobre estas são colocados pedaços de madeira e solo. No aparato da parte superior da armadilha, um pote plástico de 11 cm de largura por 10 de altura, cujo fundo foi removido, é colocado sobre o ápice da armação metálica e pelo seu interior é introduzida a manga da rede. A parte da manga que transpassa a extensão do pote é dobrada para o lado externo, e em seguida presa pela tampa oca do próprio pote. Sobre este pote, um funil, perfeitamente encaixado, conecta toda a parte inferior da armadilha a um segundo pote plástico (coletor) $\mathrm{com} 7 \mathrm{~cm}$ de largura por 7 de altura, no qual fica armazenado uma solução conservante à base de água, álcool $96 \%$, ácido acético $10 \%$ e caulim. Depois de instaladas, as armadilhas eram cobertas com sacos plásticos transparentes a fim de evitar chuva direta sobre as mesmas.
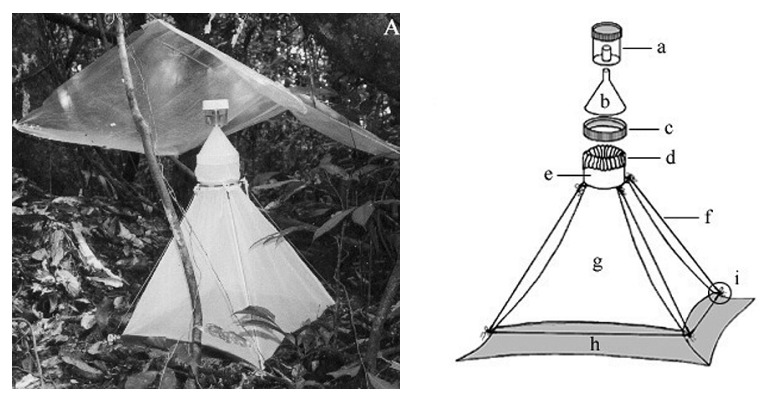

Figura 1 - A - Armadilha de emergência instalada. B - Esquema da armadilha pré-montada: $a$ - copo coletor; $b$ - funil; $c$ - tampa vazada; $d$ - manga da rede; $\mathrm{e}$ - pote; $\mathrm{f}$ - eixo de ferro; $\mathrm{g}$ - rede; $\mathrm{h}$ - aba da rede; $\mathrm{i}$ - um dos pontos de encaixe da cantoneira com os eixos de ferro.

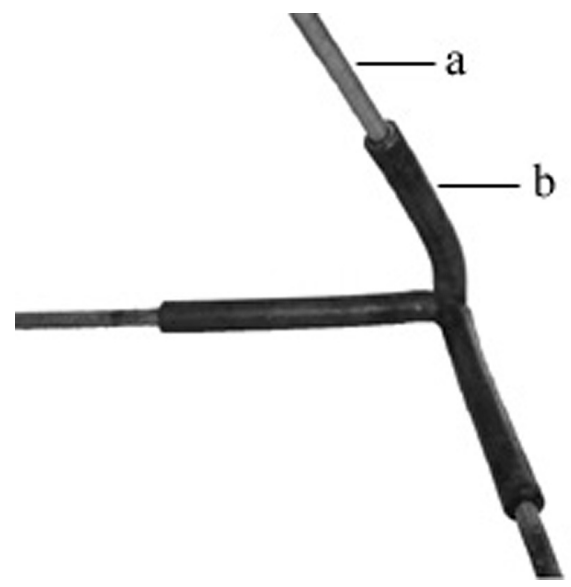

Figura 2 - Detalhe dos encaixes da cantoneira de cobre com os eixos de ferro: $a$ - eixo de ferro; $b$ - cantoneira de cobre.

\section{ÁREA DE ESTUDO}

As armadilhas foram instaladas, ao longo de três trilhas no chão de uma floresta de terra-firme localizada em torno da Vila do Pitinga (0047’28.7" S; 6004’12.2" W), município de Presidente Figueiredo, Estado do Amazonas. 


\section{CAPTURA DE FLEBOTOMINEOS}

Inicialmente, apenas 15 armadilhas foram instaladas no início da segunda quinzena do mês de junho de 2002 e permaneceram no mesmo no local por até um mês, quando então foram retiradas e instaladas em novos pontos, juntamente com mais 15 . Portanto, a partir do mês de julho de 2002 um total de 30 armadilhas se encontravam instaladas e lá permaneceram até o mês de dezembro do mesmo ano. A distância entre uma armadilha e outra subseqüente, em cada trilha, variou entre 5 a $50 \mathrm{~m}$. Todas as armadilhas foram instaladas a uma distância superior a dois metros de pontos (árvores) previamente selecionados para estudo paralelo com imaturos que se desenvolvem em bases de árvores.

Baseado no estudo do ciclo de vida de alguns flebotomíneos, em condições de laboratório, principalmente de espécies neotropicais, que apresentaram um período de fase imatura relativamente longo, normalmente superior a um mês (Johnson \& Hertig, 1961; Forattini, 1973; Ward \& Killick-Kendrick, 1974; Ward, 1977; Ferro et al, 1998; Ximenes et al, 2001; Justiniano et al., 2004), a partir de julho de 2002 , as armadilhas permaneceram em um mesmo ponto por até dois meses, sendo retiradas após este período e instaladas novamente em um ponto adjacente ao anterior.

Durante o período de julho a dezembro de 2002, quando as 30 armadilhas já se encontravam instaladas, estas foram transferidas de locais por duas vezes. No ultimo local, no entanto, permaneceram por apenas um mês. A busca do material retido no pote coletor era realizada mensalmente e este era novamente abastecido com mais solução conservante. Todo material coletado foi conservado em álcool $70 \%$ e posteriormente identificado segundo Young e Duncan (1994) e Freitas \& Barrett (2002).

\section{ANÁLISE DOS DADOS}

A produção de flebotomíneos foi baseada no modelo de cálculo utilizado por Rutledge \& Ellenwood (1975), que levaram em consideração número de flebotomíneos coletados, número de coletas diárias por armadilhas e total de área coberta por cada uma. No presente estudo, no entanto, foi considerado cada dia de permanência da armadilha no chão da floresta como sendo uma coleta diária dos adultos recém-emergidos. A área de chão de floresta coberta por cada armadilha foi de $0,25 \mathrm{~m}^{2}$.

\section{RESULTADOS}

Durante os seis meses de estudo, as armadilhas permaneceram em bom estado de conservação, com exceção do tecido que apresentou manchas provavelmente causadas por fungos. Em algumas ocasiōes, algumas armadilhas foram parcialmente danificadas por queda de galhos ou removidas por animais, sem haver, no entanto, perda do material do interior do pote coletor. Estas armadilhas foram imediatamente restauradas e repostas no seu local.
Treze das 30 armadilhas de emergência foram eficazes e apreenderam 27 flebotomíneos, numa razão de 2,08 flebotomíneos por armadilha. O número de indivíduos apreendidos por cada armadilha variou entre um e seis, a maioria com um ou dois indivíduos.

Nove espécies de flebotomíneos, todas do gênero Lutzomyia, foram encontradas e distribuídas entre quatro subgêneros (Psychodopygus, Nyssomyia, Evandromyia e Trichopygomyia) e 1 grupo (saulensis) (Tabela 1). O subgênero Psychodopygus foi o que apresentou o maior número de espécies. A espécie $L$. umbratilis do subgênero Nyssomyia, que apresentou ainda $L$. anduzei, foi a espécie mais abundante entre todas as capturadas, representando $37,1 \%$ do total de indivíduos.

Os meses de setembro e novembro foram os meses de maior e menor captura, respectivamente. No primeiro, 13 indivíduos foram capturados, sendo estes representados principalmente por L. umbratilis, enquanto no segundo foi apenas um ( $L$. monstruosa).

No presente estudo foi estimada uma produção 2,2 flebotomíneos por $100 \mathrm{~m}^{2}$ por dia. Em setembro, mês com maior número de flebotomíneos, esta produção foi de 5,8.

Tabela 1 - Espécies de flebotomíneos adultos capturados em armadilha de emergência no período de junho a dezembro/2002 em floresta de terra-firme na Vila do Pitinga-AM.

\begin{tabular}{|c|c|c|c|c|c|c|c|c|c|c|c|}
\hline \multirow{2}{*}{$\begin{array}{c}\text { Subgênero ou } \\
\text { Grupo }\end{array}$} & \multirow{2}{*}{ Espécie } & \multicolumn{6}{|c|}{$\mathrm{N}^{0}$ de indivíduos / mês } & \multicolumn{2}{|c|}{ Sexo } & \multirow{2}{*}{ Total } & \multirow{2}{*}{$\%$} \\
\hline & & J & A & S & 0 & $\mathrm{~N}$ & D & $\sigma^{\pi}$ & $q$ & & \\
\hline \multirow{3}{*}{ Psychodopygus } & L. ayrozai & 1 & 2 & 0 & 0 & 0 & 0 & 1 & 2 & 3 & 11,1 \\
\hline & L. davisi & 0 & 0 & 0 & 0 & 0 & 1 & 0 & 1 & 1 & 3,7 \\
\hline & L. geniculata & 1 & 0 & 0 & 0 & 0 & 0 & 0 & 1 & 1 & 3,7 \\
\hline \multirow{2}{*}{ Nyssomyia } & L. umbratilis & 0 & 0 & 9 & 1 & 0 & 0 & 6 & 4 & 10 & 37,1 \\
\hline & L. anduzei & 0 & 2 & 0 & 0 & 0 & 1 & 0 & 3 & 3 & 11,1 \\
\hline \multirow{2}{*}{ Evandromyia } & L. monstruosa & 0 & 0 & 3 & 0 & 1 & 0 & 0 & 4 & 4 & 14,8 \\
\hline & L. georgii & 0 & 0 & 0 & 1 & 0 & 0 & 0 & 1 & 1 & 3,7 \\
\hline \multirow{2}{*}{$\begin{array}{l}\text { Trichopygomyia } \\
\text { saulensis* }\end{array}$} & L. trichopyga & 1 & 1 & 1 & 0 & 0 & 0 & 1 & 2 & 3 & 11,1 \\
\hline & L. saulensis & 0 & 0 & 0 & 1 & 0 & 0 & 0 & 1 & 1 & 3,7 \\
\hline Total & & 3 & 5 & 13 & 3 & 1 & 2 & 8 & 19 & 27 & 100,0 \\
\hline
\end{tabular}

\section{DISCUSSÃO}

Dos quatro subgêneros e um grupo registrado no presente estudo, Psychodopygus foi o subgênero com maior número de espécies. Comparativamente, Hanson (1961) e Rutledge \& Ellenwood (1975a), ambos no Panamá, também recuperaram espécies desse mesmo subgênero exclusivamente em folhas em decomposição no chão da floresta, sugerindo que este ambiente seja o criadouro preferencial para algumas das espécies deste subgênero. 
As nove espécies registradas no presente estudo, representam aproximadamente $16 \%$ do total de espécies (55) encontradas na área de estudo, que tem como espécie mais abundante Lutzomyia umbratilis (informações baseadas em coletas de flebotomíneos realizadas no período de 1999-2000 por Raul Guerra de Queiroz / INPA, dados não publicados). Utilizando esta mesma técnica, na região de Manaus, Arias \& Freitas (1982) registraram a ocorrência de 15 das 50 espécies conhecidas na área àquela época. Das espécies coletadas por estes autores, $L$. umbratilis, $L$. anduzei e L. trichopyga, estão entres as mais representativas. Comparativamente, no presente estudo, L. umbratilise $L$. anduze $i$, também foram bem representadas, o que sugere que o chão de floresta também seja um dos principais microhabitats utilizados como criadouros por estas espécies.

A produção relativamente baixa de flebotomíneos, encontrada no presente estudo, quando comparada àquelas encontrada por Rutledge \& Ellenwood (1975a), Arias \& Freitas (1982) e Casanova (2001), 24,4; 4,1 e 24 flebotomíneos por $100 \mathrm{~m}^{2}$ por dia, respectivamente, pode não estar refletindo, mesmo entre estes autores, a eficiência da armadilha utilizada, mas sim do método (tempo de permanência da armadilha no mesmo local que, entre estes autores, variou de 14 a 39 dias). A maior extensão de área coberta pelas armadilhas, devido ao maior número de mudanças, ocasionadas pelo menor tempo de permanência destas em um mesmo local, possibilita maiores chances de captura de adultos. No entanto o maior tempo de permanência de uma armadilha em um mesmo local possibilita uma apreensão mais próxima do real de uma mesma área, já que permite a captura de espécies com ciclo de vida mais longo. Em uma das armadilhas no presente estudo, por exemplo, a captura de flebotomíneos só foi observada no segundo mês de permanência desta no mesmo local, sendo representado principalmente pela espécie $L$. umbratilis, o que sugere que esta espécie apresente um ciclo de vida bastante longo. Este dado corrobora com a maioria dos estudos sobre o período de desenvolvimento das formas imaturas de espécies neotropicais, em condiçōes de laboratório, que é em média superior a 30 dias (Johnson \& Hertig, 1961; Forattini, 1973; Ward \& Killick-Kendrick, 1974; Ward, 1977; Ferro et al., 1998; Ximenes et al., 2001; Justiniano et al., 2004).

A emergência de flebotomíneos em florestas tropicais parece está relacionada com a precipitação pluviométrica, sendo maior no período de maior precipitação (Arias \& Freitas, 1982; Ximenes et al., 2006). Curiosamente, no presente estudo, setembro, mês de baixa precipitação, foi o mês com maior número de flebotomíneos capturados. Este dado sugere que tais adultos sejam remanescentes de imaturos que se desenvolveram durante o final da estação chuvosa (Junho, Julho e Agosto). Esta informação parece está em consonância com o estudo de Ximenes et al. (2001) com Lutzomyia evandroi, onde foi observado um aumento no número de ovos postos, por fêmeas capturadas nos meses de julho e agosto, final do período chuvoso. Estes autores informam que as oviposições são mais intensas durante o período mais favorável ao desenvolvimento dos imaturos, o que inclui o final da estação de maior precipitação. No entanto, chuvas ocasionais comuns na região durante a estação de baixa pluviosidade, e observadas durante o período de estudo, podem também ter influenciado neste aumento. Obviamente, o período de estudo e o número de indivíduos coletados foi muito reduzido para permitir uma análise conclusiva a respeito de uma possível flutuação sazonal.

A formação de teias de aranha no interior das armadilhas entre uma busca e outra do material coletado, além de provável ação predatória de inimigos naturais de flebotomíneos, pode ter influenciado na produção total de flebotomíneos capturados. Apesar do uso de uma solução conservadora, que permita um período mais longo para a busca do material aprisionado no pote coletor, é aconselhável um prazo mais curto para tal busca, a fim de evitar perdas maiores.

Embora a produção de flebotomíneos tenha sido relativamente baixa, devido provavelmente aos fatores citados anteriormente (menor cobertura de área, formação de teias de aranha e provável ação predatória), a eficiência da armadilha pôde ser observada pelo grande número de outros artrópodes capturados: dípteros (além de flebotomíneos), colêmbolas, hymenopteros (vespas e formigas) entre outros.

A grande maioria das armadilhas de emergências usadas em estudos com criadouros naturais de flebotomíneos delimita a área coberta e utiliza material na sua estrutura que impede a ventilação e a entrada de luz, o que, por sua vez, pode alterar as condições naturais da área do solo coberta pela armadilha. Esta modificação do ambiente natural pode forçar a saída de larvas do interior das armadilhas ou mesmo causar a morte destas.

Uma das características da armadilha utilizada neste estudo, é que a mesma não delimita a camada do solo em profundidade, o que supostamente evitaria a saída e/ou entrada de larvas para área coberta pela armadilha. Esta não-delimitação foi proporcionada pelo tipo de tecido utilizado nas armadilhas e a cobertura feita de plástico transparente, o qual provavelmente não alteraria, ou alteraria muito pouco, $o$ ambiente natural no interior destas.

\section{AGRADECIMENTOS}

À CAPES, INPA/PPI pelo suporte financeiro, ao Sr. Josimar Pires e Dr. Oscar P. Júnior em nome da Mineração Taboca S.A. e Logos Pró-Saúde S.A., respectivamente, pelo apoio logístico em Pitinga, à Dra Sílvia Cássia B. Justiniano pela assistência durante este trabalho, ao Sr. João Vidal pelo fornecimento da solução conservante, ao Sr. Walter Santos pela ajuda na confecção das armadilhas, ao técnico Raimundo Nonato pelo auxílio no campo e aos Srs. Nunes e Mendes pelo apoio técnico em Pitinga. 


\section{BIBLIOGRAFIA CITADA}

Arias, J.R; Freitas, R.A. 1982. On the vectors of cutaneous leishmaniasis in the Central Amazon of Brazil. 4. Sand fly emergence from a "terra firme" forest floor. Acta Amazonica, 12(3): 609-611.

Bettini, S.; Contini, C.; Atzeni, M.C.; Tocco, G. 1986. Leishmaniasis in Sardinia. I. Observations on a larval breeding site of Phlebotomus perniciosus, Phlebotomus perfiliewi perfiliewi and Sergentomyia minuta (Diptera: Psychodidae) in the canine leishmaniasis focus of Soleminis (Cagliari). Annals of Tropical Medicine and Parasitology, 80(3): 307-315.

Casanova, C. 2001. A Soil Emergence Trap for Collections of Phlebotomine Sand Flies. Memórias do Instituto Oswaldo Cruz, 96(2): 273-275.

Feliciangeli, M.D. 2004. Natural breeding places of phlebotomine sandflies. Medical and Veterinary Entomology, 18: 71-80.

Ferro, C.; Cárdenas, E.; Corredor, D.; Morales, A.; Munstermann, L.E. 1998. Life Cycle and Fecundity Analysis of Lutzomyia shannoni (Dyar) (Diptera: Psychodidae) Memórias do Instituto Oswaldo Cruz, 93(2): 195-199.

Forattini, O.P. 1973. Entomologia médica, Vol. IV, Editora Edgar Blücher, São Paulo, Brasil, 658pp.

Freitas, R.A.; Barrett, T.V. 2002. Description of Luzomyia (Evandromyia) georgii n. sp. and a Synopsis of the Series infraspinosa (Diptera: Psychodidae). Memórias do Instituto Oswaldo Cruz, 97(2): 239-245.

Hanson, W.J. 1961. The breeding places of Phlebotomus in Panama (Diptera: Psychodidae). Annals of the Entomological Society of America, 54: 317-322.

Johnson, P.T.; Hertig, M. 1961. The rearing of Phlebotomus sandflies (Diptera: Psychodidae). II - Development and behavior of Panamanian sandflies in laboratory culture. Annals of the Entomological Society of America, 54: 764-776.

Justiniano, S.C.B.; Chagas, A.C.; Pessoa, F.A.C.; Queiroz, R.G. 2004. Comparative biology of two populations of Lutzomyia umbratilis (Diptera: Psychodidae) of Central Amazonia, Brazil, under laboratory conditions. Brazilian Journal of Biology, 64(2): 227235 .
Penny, N.D.; Arias, J.R. 1982. Insect of an Amazon Forest, Columbia University Press, New York, United States of America, 269pp.

Rutledge, L.C.; Ellenwood D.A. 1975. Production of phlebotomine sandflies on the open forest floor in Panama: The species complement. Environmental Entomology, 4: 71-77.

Young, D.G; Duncan, M.A. 1994. Guide to the Identification and Geographic Distribution of Lutzomyia Sand Flies in Mexico, the West Indies, Central and South America (Diptera: Psychodiadae). Memoirs of the American Entomological Institute. Associated Publishers, Gainesville, USA. vol. 54, 881pp.

Ximenes, M.F.F.M.; Maciel, J.C.; Jerônimo, S.M.B. 2001 Characteristics of the Biological Cycle of Lutzomyia evandroi Costa Lima \& Antunes, 1936 (Diptera: Psychodidae) under Experimental Conditions. Memórias do Instituto Oswaldo Cruz, 96(6): 883-886

Ximenes, M.F.F.M.; Castellón, E.G.; Souza, M.F.; Menezes, A.A.L.; Queiroz, J.W.; Silva, V.P.M.; Jerônimo, S.M.B. 2006. The Effect of Abiotic Factors on Seasonal Population Dynamics of Lutzomyia longipalpis (Diptera: Psychodidae) in Northeastern Brazil. Journal of Medical Entomology, 43: 990-995.

Ward, R.D. 1977. The colonization of Lutzomyia flaviscutellata (Diptera: Psychodidae), a vector of Leishmania mexicana amazonensis in Brazil. Journal of Medical Entomology, 4(4): 469476.

Ward, R.D.; Killick-Kendrick, R. 1974. Field and laboratory observations on Psychodopygus lainsoni Fraiha \& Ward and other sandflies (Diptera, Phlebotomidae) from the Transamazônica high-way, Pará State, Brazil. Bulletin of Entomological Research, 64: 213-211.

Recebido em 16/10/2006

Aceito em 10/04/2007 
